\title{
Nonlinear decay of a large-ampitude ion-acoustic wave
}

\section{BY NGUYEN THE HUNG}

Ecole Polytechnique Fédérale de Lausanne, Switzerland, Centre de Recherches en Physique des Plasmas

(Received 13 January 1975 and in revised form 16 June 1975)

This paper studies the nonlinear coupling between an ion-acoustic wave and two Alfvén waves. On the basis of the MHD equations, this three-wave interaction is described within the coupled normal-mode theory. It is shown that a largeamplitude acoustic wave can decay into a pair of Alfvén waves in a high- $\beta$ plasma. Calculations of the threshold condition and the maximum growth rate suggest that this decay process may occur both in the laboratory and in the ionosphere.

\section{Introduction}

Under various conditions, a plasma can become unstable against the buildup of large-amplitude ion-acoustic waves. So far, nonlinear studies of such waves have been focussed on the effects of self-coupling (harmonic generation, amplitude modulation) and wave-particle interaction (particle trapping). Nonlinear decay of a finite-amplitude acoustic wave, to our knowledge, has not been investigated. The purpose of this paper is, accordingly, to study the coupling between ionacoustic and Alfvén waves in a high- $\beta$ plasma.

In $\S 2$ a set of coupled equations for the waves under consideration is obtained, using a fluid description. In $\S 3$ it will be shown that, when the sound velocity is greater than the Alfvén velocity, a large-amplitude acoustic wave, acting as a pump, can decay parametrically into a pair of Alfvén waves. The physical mechanism for this decay process can be described as follows. At frequencies well below the ion-cyclotron frequency, the plasma is frozen in the magnetic field lines. Accordingly, the Alfvén waves can be considered as the transverse motion of these field lines, in which the magnetic pressure provides the restoring force, while the plasma mass acts as an inertial force. In fact, Alfvén waves are simply the transverse waves on a string of tension $H_{0}^{2} / 4 \pi$ and mass density $\rho_{0}$. Thus, by modulating the mass density of the plasma along the magnetic field lines, an acoustic wave just provides the necessary mechanism for the parametric excitation of these Alfvén waves. As we shall see, this process requires a rather low threshold, and can occur both in laboratory plasma (e.g. pinch plasmas) and in the ionosphere (solar wind). 


\section{The coupled equations}

Let the equilibrium state of a uniform, unbounded plasma be characterized by a mass density $\rho_{0}$, a pressure $p_{0}$, and a steady magnetic field $\mathbf{H}_{0}=H_{0} \mathbf{z}$. On the basis of the MHD equations, we shall consider the interaction between an acoustic wave and two Alfvén waves (denoted by indices 1 and 2), all propagating along the magnetic field.

By neglecting terms corresponding to the self-coupling of these waves, the governing nonlinear equations can be expressed as

$$
\begin{gathered}
\frac{\partial \rho}{\partial t}+\rho_{0} \frac{\partial v}{\partial z}=0 \\
\frac{\partial v}{\partial t}+\frac{c_{S}^{2}}{\rho_{0}} \frac{\partial \rho}{\partial z}+\nu v=-\frac{1}{4 \pi \rho_{0}} \frac{\partial}{\partial z}\left(H_{1} H_{2}\right) \equiv P \\
\frac{\partial H_{1,2}}{\partial t}-H_{0} \frac{\partial V_{1,2}}{\partial z}-\eta \frac{\partial^{2} H_{1,2}}{\partial z^{2}}=-\frac{\partial}{\partial z}\left(v H_{2,1}\right) \equiv Q_{1,2} \\
\frac{\partial V_{1,2}}{\partial t}-\frac{c_{A}^{2}}{H_{0}} \frac{\partial H_{1,2}}{\partial z}=-v \frac{\partial V_{2,1}}{\partial z}-\frac{c_{A}^{2}}{\rho_{0} H_{0}}\left(\rho \frac{\partial H_{2,1}}{\partial z}\right) \equiv R_{1,2} .
\end{gathered}
$$

Here, $\rho$ and $v$ are the perturbed density and velocity due to the acoustic waves, $H_{1,2}$ and $V_{1,2}$ are the perturbed magnetic fields and velocities associated with the Alfvén waves $(1,2) . c_{S}$ and $c_{A}$ are the velocities of the acoustic and Alfvén waves, respectively. $\nu$ represents the phenomenological damping coefficient for the ion wave, while $\eta$ is the plasma resistivity.

In linear theory, the right-hand sides of $(1 a-d)$ obviously vanish. As it stands, $(1 a-d)$ is a formidable nonlinear system, whose solutions can be sought only within various approximations. For a low- $\beta$ plasma $\left(c_{S} \ll c_{A}\right)$, one can neglect the first terms on the right-hand sides of $(1 c, d)$ because of their smallness compared to the second terms. In this case, Sagdeev \& Galeev (1969) have found that a large-amplitude Alfvén wave can decay into a daughter Alfvén wave and an acoustic wave. Hung (1974) showed that, in fact, an Alfvén wave can give rise to two distinct types of parametric instabilities, namely the oscillating and the purely growing waves.

In the case of high- $\beta$ plasmas $\left(c_{A}<c_{S}\right)$, one has not only to keep all the terms on the right-hand sides of $(1 c, d)$, but also to note that the foregoing decay process is no longer possible. However, it is now the acoustic wave that will decay into a pair of Alfvén waves.

This phenomenon can be described by various methods. Here, the coupledmode theory will lead in a simple way to a set of first-order differential equations, and show clearly the coupling mechanism of the waves under consideration. Let us first introduce the eigenfunctions of (1) defined as (Hung 1974)

$$
a=\rho+c_{1} v, \quad b=H+c_{2} V .
$$

The coefficients $c_{1}$ and $c_{2}$ are chosen so that, in the linear limit,

$$
\frac{\partial a}{\partial t}+\gamma a=-i \omega_{S} a, \frac{\partial b}{\partial t}+\Gamma b=-i \omega_{A} b .
$$


$\omega_{S}, \omega_{A}, \gamma$ and $\Gamma$ are the frequencies and linear damping rates of the acoustic and Alfvén waves, respectively.

It is simple to show that, for a given wave vector $\mathbf{k}_{S}$, there exist two eigenfunctions of the sound wave (Hung 1974)

$$
a^{ \pm}=\rho^{ \pm} \pm\left(\rho_{0} k_{S} / \omega_{S}\right) v \pm \sim \exp \left\{i k_{S} z \mp i \omega_{S} t-\gamma t\right\}
$$

with

$$
\omega_{S}=\left|c_{S} k_{S}\right|, \quad \gamma=\frac{1}{2} \nu \text {. }
$$

Obviously, $a^{+}$and $a^{-}$correspond to the forward and backward propagating waves with respect to the wave vector $\mathbf{k}_{S}$. For the Alfvén waves, we obtained

$$
b_{1,2}^{ \pm}=H_{1,2}^{ \pm} \mp\left(H_{0} k_{1,2} / \omega_{1,2}\right) V_{1,2}^{ \pm} \sim \exp \left\{i k_{1,2} z \mp i \omega_{1,2} t-\Gamma_{1,2} t\right\},
$$

with

$$
\omega_{1,2}=\left|c_{A} k_{1,2}\right|, \quad \Gamma_{1,2}=\frac{1}{2} \eta k_{1,2}^{2} .
$$

The perturbed quantities $\rho, v, V$ and $H$ can be expressed in terms of these eigenfunctions by the relations

$$
\rho^{ \pm}=\frac{1}{2} a^{ \pm}, \quad v^{ \pm}= \pm \frac{\omega_{S}}{2 \rho_{0} k_{S}} a^{ \pm}, \quad V_{1,2}^{ \pm}=\frac{\omega_{1,2}}{2 H_{0} k_{1,2}} b_{1,2}^{ \pm}, \quad H_{1,2}^{ \pm}=\frac{1}{2} b_{1,2}^{ \pm} .
$$

It is now easy to show that $(1 a-d)$ is equivalent to

$$
\left.\begin{array}{c}
\frac{\partial a^{ \pm}}{\partial t} \pm c_{S} \frac{\partial a^{ \pm}}{\partial z}+\gamma a \pm=\frac{\rho_{0} k_{s}}{\omega_{s}} P, \\
\frac{\partial b_{1}^{ \pm}}{\partial t} \pm c_{A} \frac{\partial b_{1}^{ \pm}}{\partial z}+\Gamma_{1} b_{1}^{ \pm}=Q_{1} \mp \frac{H_{0} k_{1}}{\omega_{1}} R_{1} \\
\frac{\partial b_{\frac{1}{2}}^{ \pm}}{\partial t} \pm c_{A} \frac{\partial b_{2}^{ \pm}}{\partial z}+\Gamma_{2} b_{2}^{ \pm}=Q_{2} \mp \frac{H_{0} k_{2}}{\omega_{2}} R_{2}
\end{array}\right\}
$$

$P, Q, R$ are expressed in terms of the eigenfunctions $a, b$ through (6). In a weakcoupling process, (7) can be readily solved by assuming solutions of the form

$$
\left.\begin{array}{rl}
a \pm & =A \pm(t) \exp \left\{i k_{\mathrm{S}} z \mp i \omega_{\mathrm{S}} t\right\}+\text { complex conjugate, } \\
b_{1,2}^{ \pm} & =B_{1,2}^{ \pm}(t) \exp \left\{i k_{1,2} z \mp i \omega_{1,2} t\right\}+\text { complex conjugate, }
\end{array}\right\}
$$

with

$$
\frac{d}{d t}|\ln A \pm| \ll \omega_{s}, \quad \frac{d}{d t}\left|\ln B_{1,2}^{ \pm}\right| \ll \omega_{1,2} .
$$

Then, keeping only terms with approximately the same oscillating exponentials on both sides of (7) and taking a space average of the resulting equations, we finally arrive at the following coupled system for the amplitudes of the waves under consideration:

$$
\left.\begin{array}{l}
\frac{\partial A^{+}}{\partial t}+\gamma A^{+}=-\frac{i k_{S}^{2}}{16 \pi \omega_{s}} B_{1}^{+} B_{2}^{+} \exp \{i \delta t\}, \\
\frac{\partial B_{1}^{+}}{\partial t}+\Gamma_{1} B_{1}^{+}=\frac{i}{4 \rho_{0}}\left(k_{2} c_{A}-k_{S} c_{S}\right) A^{+} \bar{B}_{2}^{+} \exp \{-i \delta t\}, \\
\frac{\partial B_{2}^{+}}{\partial t}+\Gamma_{2} B_{2}^{+}=-\frac{i}{4 \rho_{0}}\left(k_{1} c_{A}+k_{S} c_{S}\right) A^{+} \bar{B}_{1}^{+} \exp \{-i \delta t\} .
\end{array}\right\}
$$

(Similar equations can be obtained for the backward waves which, however, do not interfere with the forward waves in the case $\omega_{1}, \omega_{2} \gg \delta$ considered here.) 
Note that, in obtaining (9), use has been made of the matching condition

$$
\mathbf{k}_{S}=\mathbf{k}_{1}+\mathbf{k}_{2}, \quad \omega_{S}=\omega_{1}+\omega_{2}+\delta, \quad \delta \ll \omega_{1}, \omega_{2} .
$$

Thus, (9) represents the resonant coupling between three waves treated on the same footing. In $\S 3$ we shall study the case of parametric coupling, in which one of the waves has an amplitude much larger than the others, and can be considered as constant.

\section{The decay of a large-amplitude acoustic wave}

In many cases, a large-amplitude acoustic wave can be generated either by the plasma itself, or by some external source. Nonlinear effects then play an important role in its evolution. On the one hand, wave-particle interactions will lead the wave-plasma system to a quasi-steady state. On the other hand, the wave may decay into a pair of Alfvén waves through the resonant interaction described above. Conditions for this decay process, and growth rates of the excited waves can be determined from (9), considering the acoustic wave as a pump wave of constant power.

By introducing the variables

$$
x=B_{1}^{+}, \quad y=\bar{B}_{2}^{+} \exp \{-i \delta t\}
$$

in (9), and assuming solutions of the form $x, y \sim \exp \{-i \omega t\}$, we readily obtain

$$
\left.\begin{array}{r}
\left(-i \omega+\Gamma_{1}\right) x=\left(i / 4 \rho_{0}\right)\left(k_{2} c_{A}-k_{S} c_{S}\right) A^{+} y, \\
\left(-i \omega+i \delta+\Gamma_{2}\right) y=\left(i / 4 \rho_{0}\right)\left(k_{1} c_{A}+k_{S} c_{S}\right) \bar{A}^{+} x
\end{array}\right\}
$$

This yields the dispersion relation

$$
\omega^{2}+i\left(\Gamma_{1}+\Gamma_{2}+i \delta\right) \omega=\Gamma_{1}\left(\Gamma_{2}+i \delta\right)-\left(\omega_{S}-\omega_{1}\right)\left(\omega_{S}-\omega_{2}\right) \frac{|A|^{2}}{16 \rho_{0}^{2}}
$$

From (13), it is easily shown that growing solutions exist if

$$
|A|^{2}>\frac{16 \rho_{0}^{2} \Gamma_{1} \Gamma_{2}}{\left(\omega_{S}-\omega_{1}\right)\left(\omega_{S}-\omega_{2}\right)}\left[1+\frac{\delta^{2}}{\left(\Gamma_{1}+\Gamma_{2}\right)^{2}}\right] .
$$

Equation (14) is just the threshold amplitude for the decay of an acoustic wave into a pair of Alfvén waves.

Under the perfect matching condition, the threshold amplitude assumes its minimum value

or

$$
\begin{aligned}
& A_{m}=4 \rho_{0}\left(\Gamma_{1} \Gamma_{2} / \omega_{1} \omega_{2}\right)^{\frac{1}{2}}, \\
& \rho_{m}=\rho_{0} \eta\left(\omega_{S} / c_{A}^{2}\right)\left(1-c_{A}^{2} / c_{S}^{2}\right)^{\frac{1}{2}},
\end{aligned}
$$

if use is made of the linear dispersion relations (4)-(5), together with the resonant condition (10). Above this minimum threshold, both Alfvén waves are excited with a growth rate

$$
\Gamma_{m}=-\frac{1}{2}\left(\Gamma_{1}+\Gamma_{2}\right)+\frac{1}{2}\left[\left(\Gamma_{1}-\Gamma_{2}\right)^{2}+\rho^{2} \omega_{1} \omega_{2} / \rho_{0}^{2}\right]^{\frac{1}{2}} .
$$

Well above threshold, we have

$$
\Gamma_{m}=\left(\rho \omega_{S} / 8 \rho_{0}\right)\left(1-c_{A}^{2} / c_{S}^{2}\right)^{\frac{1}{2}}
$$


For $c_{S}=2 c_{A}$, and an average damping rate $\left(\Gamma_{1} \Gamma_{2} / \omega_{1} \omega_{2}\right)^{\frac{7}{2}}=10^{-3}$, these formulae predict a minimum threshold $\rho_{m} / \rho_{0}=4 \times 10^{-3}$ and a maximum growth rate $\Gamma_{m} / \omega_{s} \simeq \rho / 10 \rho_{0}$.

From these results, it appears that the threshold amplitude required for the parametric decay of an acoustic wave is rather low, while the growth rate of the excited Alfvén waves is directly proportional to the modulation density. Consequently, we believe that this decay process can be easily observed both in the laboratory (e.g. in high- $\beta$ pinch plasmas) and in the ionosphere. In this latter case, it provides an alternative explanation for the emission of Alfvén waves in the solar wind, when the temperature anisotropy is too weak to excite the fire-hose instability (Kennel \& Scarf 1969).

Finally, it is interesting that the high- $\beta$ limits $\left(c_{A} \ll c_{S}\right)$ of our (15) and (17) (minimum threshold and maximum growth rate) are identical with the results of Vahala \& Montgomery (1971), and Lashmore-Davies \& Ong (1974), in the opposite limit. In fact, these analyses apply to the experimental situation of Lehane \& Paoloni (1972), where Alfvén waves were parametrically excited by modulating the background magnetic field in a low- $\beta$ plasma $\left(c_{A} \gg c_{S}\right)$.

The author thanks Dr F. Hofmann for reading the manuscript. This work was performed under the auspices of the Swiss National Science Foundation.

\section{REFERENCES}

Kennel, C. F. \& Scarf, F. L. 1969 Plasma Waves in Space and in the Laboratory. Edinburgh University Press.

Lashmore-Davies, C. N. \& ONG, R. S. B. 1974 Phys. Rev. Letters, 32, 1172.

Lehane, J. A. \& Paolont, F. J. 1972 Plasma Phys. 14, 461.

Hung, N. T. 1974 J. Plasma Phys. 12, 445.

Sagdeev, R. Z. \& Gareev, A. A. 1969 Nonlinear Plasma Theory. Benjamin.

Vahala, G. \& Montgomery, D. 1971 Phys. Fluids, 14, 1137. 